\title{
Effect of chemical reaction on MHD flow with heat and mass transfer past a vertical porousplate in the presence of viscous dissipation
}

\author{
S. Kar ${ }^{1}$, N. Senapati², B. K. Swain ${ }^{3}$ \\ 1,2 Department of Mathematics, Ravenshaw University, India \\ ${ }^{3}$ Indira Gandhi Institute of Technology, India
}

\begin{tabular}{l}
\hline \hline Article Info \\
\hline Article history: \\
Received Dec 9, 2018 \\
Revised Feb 7, 2019 \\
Accepted Feb 21, 2019 \\
\hline Keywords: \\
Chemical reaction \\
Eckert number \\
MHD \\
Soret number \\
Viscous dissipation \\
\hline
\end{tabular}

\begin{abstract}
An attempt is made to study an unsteady MHD free convective flow with heat and mass transfer past a semi-infinite vertical porous plate immersed in a porous medium. Presence of viscous dissipation and chemical reaction are taken into account. It is assumed that the plate is moved with uniform velocity in the direction of fluid flow. Viscous dissipation term leads nonlinearity in the governing equations. Applying perturbation technique, the solutions for velocity, temperature and concentration are obtained. The effect of various parameters such as Rc, Gr, Gc, Sc etc. On velocity, temperature and concentration are shown through graphs.
\end{abstract}

Corresponding Author:

Satyabrat Kar,

Department of Mathematics,

Ravenshaw University,

753003 Cuttack, Odisha, India.

Email: satyabratkr@gmail.com

\section{NOMENCLATURE}

$C^{*} \quad$ Species concentration $\left(\mathrm{kg} \mathrm{m}^{-3}\right)$

$C_{p} \quad$ Specific heat at constant pressure $\left(\mathrm{Jkg}^{-1} \mathrm{~K}\right)$

$C_{\infty}^{*} \quad$ Species concentration in the free stream $\left(\mathrm{kg} \mathrm{m}^{-3}\right)$

$C_{w}^{*} \quad$ Species concentration at the surface $\left(\mathrm{kg} \mathrm{m}^{-3}\right)$

$D_{M} \quad$ Chemical molecular diffusivity $\left(m^{2} s^{-1}\right)$

$g \quad$ Acceleration due to gravity $\left(m s^{-2}\right)$

Gr Thermal Grashof number

Gc Mass Grashof number

K Permeability parameter

M Hartmann number

$\mathrm{Nu} \quad$ Nusselt number

$\mathrm{Pr} \quad$ Prandtl number

$q_{r} \quad$ Radiative heat flux

Sh Sherwood number

Sc Schmidt number

$T^{*} \quad$ Temperature $(\mathrm{K})$

$T_{w}^{*} \quad$ Fluid temperature at the surface $(\mathrm{K})$

$T_{\infty}^{*} \quad$ Fluid temperature in the free stream $(\mathrm{K})$ 
$\mathrm{u} \quad$ Dimensionless velocity component $\left(m s^{-1}\right)$

Greek symbols

$\beta \quad$ Coefficient of volume expansion for heat transfer $\left(K^{-1}\right)$

$\beta^{\prime} \quad$ Coefficient of volume expansion for mass transfer $\left(K^{-1}\right)$

$\theta \quad$ Dimensionless fluid temperature $(\mathrm{K})$

$k \quad$ Thermal conductivity $\left(W m^{-1} K^{-1}\right)$

$v \quad$ Kinematic viscosity $\left(m^{2} s^{-1}\right)$

$\rho \quad$ Density $\left(\mathrm{kgm}^{-3}\right)$

$\sigma \quad$ Electrical conductivity

$C \quad$ Dimensionless species concentration $\left(\mathrm{kgm}^{-3}\right)$

$\tau \quad$ Shearing stress $\left(\mathrm{Nm}^{-2}\right)$

Subscripts

$w$ Conditions on the wall

$\infty$ Free stream condition

\section{INTRODUCTION}

In recent years, the subject of magnetohydrodynamics has attracted the attention of many authors in view of its application to the problems in geophysics, astrophysics and many engineering and industrial applications, like cooling of metal in nuclear reactors and magnetic control of iron flow in steel industry, etc. [1-4]. The investigation of unsteady natural convective flow of viscous incompressible fluid past vertical bodies has also several engineering and technological applications. Gupta [5] studied transient free convection of an electrically conducting fluid from a vertical plate in the presence of magnetic field. Free convection effects on flow past an exponentially accelerated vertical plate was studied by Singh and Kumar [6]. Jha et al. [7] analyzed mass transfer effects on exponentially accelerated infinite vertical plate with constant heat flux and uniform mass diffusion. Recently Ahmmed et.al. [8] studied the unsteady MHD free convection and mass transfer flow past a vertical porous plate.

Chemical reactions happen at a characteristic reaction rate at a given temperature and chemical concentration. Different chemical reactions ae used in combinations during chemical synthesis in order to find a desired product. In biochemistry, a consecutive series of chemical reactions form metabolic path ways. The order of chemical reaction is defined as the sum of the powers of the concentration of the reactants in the rate equation of that particular chemical reaction. A first order reaction is the one in which the rate is proportional to concentration of a single reactant. In the present paper, first order reaction is taken into acount. Moreover, coupled heat and mass transfer problems in the presence of chemical reaction are of importance in many processes such as drying, distribution of temperature and moisture over agricultural fields and groves of fruit trees, damage of crops due to freezing, evaporation at the surface of a water body and energy transfer in a wet cooling tower, and flow in a desert cooler. Chamkha and Aly [9] obtained numerical solution of steady boundary-layer stagnation point flow of a polar fluid toward a stretching surface embedded in porous media in the presence of the effects of Soret and Dufour numbers and first-order homogeneous chemical reaction. Aurangzaib et al. [10] investigated the effect of thermal stratification and chemical reaction on free convection boundary layer MHD flow with heat and mass transfer of an electrically conducting fluid over time dependent stretching sheet. Abd El-Aziz [11] obtained numerical results to study the effect of time dependent chemical reaction on stagnation point flow and heat transfer of nanofluid over a stretching sheet. Pal and Mandal [12,13] investigated the mixed convection boundary layer flow of nanofluids at a stagnationpoint over a permeable stretching/shrinking sheet subject to thermal radiation, heat source/sink, viscous dissipation and chemical reaction using numerical method.

In the physical realm, many irriversible processes are present. Some exmples are heat flow through a thermal resistance, fluid flow through a flow resistance, chemical reactions etc.. The irreversible process by means of which the work done by a fluid on adjacent layers due to the action of shear forces is transformed into heat is defined as viscous dissipation. It can be seen in many places such as in hydraulic engineering, waves or oscillations etc. viscous dissipation has different applications in various industries. Significant temperature rises are observed in polymer processing flows such as injection molding or extrusion at high rates. Aerodynamic heating in the thin boundary layer around high speed aircraft raises the temperature of the skin. A number of authors have considered viscous heating effects on Newtonian flows. Mahajan et al. [14] reported the influence of viscous heating dissipation effects in natural convective flows, showing that the heat transfer rates are reduced by an increase in the dissipation parameter. Isreal Cookey et al. [15] investigated the influence of viscous dissipation and radiation on unsteady MHD free convection flow past an infinite

Int. J. of Adv. in Appl. Sci. Vol. 8, No. 1, March 2019: 83 - 94 
heated vertical plate in a porous medium with time dependent suction. Zueco [16] used network simulation method (NSM) to study the effects of viscous dissipation and radiation on unsteady MHD free convection flow past a vertical porous plate. Suneetha et al. [17] have analyzed the thermal radiation effects on hydromagnetic free convection flow past an impulsively started vertical plate with variable surface temperature and concentration by taking into account of the heat due to viscous dissipation. Recently Suneetha et al. [18] studied the effects of thermal radiation on the natural conductive heat and mass transfer of a viscous incompressible gray absorbing-emitting fluid flowing past an impulsively started moving vertical plate with viscous dissipation. Very recently, Hiteesh [19] studied the boundary layer steady flow and heat transfer of a viscous incompressible fluid due to a stretching plate with viscous dissipation effect in the presence of a transverse magnetic field.

Due to above significant role, it is the purpose of this paper to examine the effect of viscous dissipation and chemical reaction on the MHD flow of a viscous incompressible fluid past a semi-infinite vertical plate. This flow problem was previously studied by Ahmmed et.al [8] in the absence of these two parameters.

\section{MATHEMATICHAL FORMULATION}

A two dimensional unsteady flow of a laminar and incompressible fluid past a semi-in finite vertical moving plate embedded in a uniform porous medium is considered. Fluid is assumed to be viscous and electrically conducting. A uniform transverse magnetic field $B_{0}$ is applied in the presence of pressure gradient. Thermal diffusion and thermal radiation are also considered to be present. In the given system $x$, axis is taken along the plate and $y^{\prime}$ axis normal to it. No voltage is applied to the system and induced magnetic field is negligible. Since we assume a semi-infinite plate surface, the flow variables are the functions of $y$ 'and $t$ ' only. Then under the above assumption the unsteady flow with usual Boussinesq's approximation is governed by the following equations.

$$
\begin{aligned}
& \frac{\partial v^{\prime}}{\partial y^{\prime}}=0 \\
& \rho\left(\frac{\partial u^{\prime}}{\partial t^{\prime}}+v^{\prime} \frac{\partial u^{\prime}}{\partial y^{\prime}}\right)=\frac{\partial p^{\prime}}{\partial x^{\prime}}+\mu \frac{\partial^{z} u^{\prime}}{\partial y^{r 2}}-\rho g-\frac{\mu}{k^{\prime}} u^{\prime}-\sigma \beta_{0}^{2} u^{\prime} \\
& \frac{\partial \mathrm{T}^{\prime}}{\partial \tau^{\prime}}+\mathrm{v}^{\prime} \frac{\partial \mathrm{T}^{\prime}}{\partial \mathrm{y}^{\prime}}=\frac{\mathrm{k}}{\rho \mathrm{C}_{\mathrm{p}}} \frac{\partial^{2} \mathrm{~T}^{\prime}}{\partial \mathrm{y}^{\prime 2}}-\frac{1}{\rho \mathrm{C}_{\mathrm{p}}}\left(\frac{\partial \mathrm{q}_{\mathrm{r}}^{\prime}}{\partial \mathrm{y}^{\prime}}\right)-\frac{\mathrm{Q}_{0}}{\rho \mathrm{C}_{\mathrm{P}}}\left(\mathrm{T}^{\prime}-\mathrm{T}_{\infty}^{\prime}\right)+\frac{\mu}{\rho \mathrm{C}_{\mathrm{p}}}\left(\frac{\partial \mathrm{u}^{\prime}}{\partial \mathrm{y}^{\prime}}\right)^{2} \\
& \frac{\partial \varphi^{\prime}}{\partial \mathrm{t}^{\prime}}+\mathrm{v}^{\prime} \frac{\partial \varphi^{\prime}}{\partial \mathrm{y}^{\prime}}=\mathrm{D}_{\mathrm{M}} \frac{\partial^{2} \varphi^{\prime}}{\partial \mathrm{y}^{\prime 2}}+\mathrm{D}_{\mathrm{T}} \frac{\partial^{2} \mathrm{~T}^{\prime}}{\partial \mathrm{y}^{\prime 2}}-\mathrm{R}_{\mathrm{C}}^{\prime}\left(\mathrm{C}^{\prime}-\mathrm{C}_{\infty}^{\prime}\right)
\end{aligned}
$$

Boundary conditions for the velocity, temperature and concentration fields are given as follows

$$
\begin{aligned}
& \mathrm{u}^{\prime}=\mathrm{u}_{\underline{\mathrm{p}}}^{\prime}, \mathrm{T}^{\prime}=\mathrm{T}_{\mathrm{w}}^{\prime}+\in\left(\mathrm{T}_{\mathrm{w}}^{\prime}-\mathrm{T}_{\infty}^{\prime}\right) \mathrm{e}^{\mathrm{n}^{\prime} \mathrm{t}^{\prime},} \mathrm{C}^{\prime}=\mathrm{C}_{\mathrm{w}}^{\prime}+\in\left(\mathrm{C}_{\mathrm{w}}^{\prime}-\mathrm{C}_{\infty}^{\prime}\right) \text { at } y^{\prime}=0 \\
& \mathrm{u}^{\prime}=\mathrm{U}_{\infty}^{\prime}, \mathrm{U}_{\infty}^{\prime}=\mathrm{U}_{0}\left(1+\epsilon \mathrm{e}^{\mathrm{n}^{\prime} \mathrm{t}^{\prime}}\right), \mathrm{T}^{\prime} \rightarrow \mathrm{T}_{\infty}^{\prime}, \mathrm{C}^{\prime} \rightarrow \mathrm{C}_{\infty}^{\prime} \text { at } y^{\prime}=\infty
\end{aligned}
$$

Now, $v^{\prime}=-v_{0}\left(1+\in A e^{n^{\prime} t^{\prime}}\right)$

in free stream, we have

$$
\begin{aligned}
& \rho \frac{\mathrm{dU}_{\infty}^{\prime}}{\mathrm{dt}^{\prime}}=\frac{\partial \mathrm{p}^{\prime}}{\partial \mathrm{x}^{\prime}}-\rho_{\infty} \mathrm{g}-\frac{\mu}{\mathrm{k}^{\prime}} \mathrm{U}_{\infty}^{\prime}-\sigma \mathrm{B}_{0}^{2} \mathrm{U}_{\infty}^{\prime} \\
& \frac{\partial \mathrm{p}^{\prime}}{\partial \mathrm{x}^{\prime}}=\rho \frac{\mathrm{dU}_{\infty}^{\prime}}{\mathrm{dt}^{\prime}}+\rho_{\infty} \mathrm{g}+\frac{\mu}{\mathrm{k}^{\prime}} \mathrm{U}_{\infty}^{\prime}+\sigma \mathrm{B}_{0}^{2} \mathrm{U}_{\infty}^{\prime}
\end{aligned}
$$

Eliminating $\frac{\partial p^{\prime}}{\partial x}$ using (2) and (8), we obtain 


$$
\begin{aligned}
\rho\left(\frac{\partial \mathrm{u}^{\prime}}{\partial \mathrm{t}^{\prime}}+\mathrm{v}^{\prime} \frac{\partial \mathrm{u}^{\prime}}{\partial \mathrm{y}^{\prime}}\right) & =\rho \frac{\mathrm{dU}_{\infty}^{\prime}}{\mathrm{dt}^{\prime}}+\rho_{\infty} \mathrm{g}+\frac{\mu}{\mathrm{k}^{\prime}} \mathrm{U}_{\infty}^{\prime}+\sigma \mathrm{B}_{0}^{2} \mathrm{U}_{\infty}^{\prime}+\mu \frac{\partial^{2} \mathrm{u}^{\prime}}{\partial \mathrm{y}^{\prime 2}}-\rho \mathrm{g}-\frac{\mu}{\mathrm{k}^{\prime}} \mathrm{u}^{\prime}-\sigma \mathrm{B}_{0}^{2} \mathrm{u}^{\prime} \\
& =\rho \frac{\mathrm{dU}_{\infty}^{\prime}}{\mathrm{dt^{ \prime }}}+\left(\rho_{\infty}-\rho\right) \mathrm{g}+\mu \frac{\partial^{2} \mathrm{u}^{\prime}}{\partial \mathrm{y}^{\prime 2}}+\frac{\mu}{\mathrm{k}^{\prime}}\left(\mathrm{U}_{\infty}^{\prime}-\mathrm{u}^{\prime}\right)+\sigma \mathrm{B}_{0}^{2}\left(\mathrm{U}_{\infty}^{\prime}-\mathrm{u}^{\prime}\right)
\end{aligned}
$$

By using the equation state, we obtain

$$
\left(\rho_{\infty}-\rho\right)=\beta\left(\mathrm{T}^{\prime}-\mathrm{T}_{\infty}^{\prime}\right)+\beta^{\prime}\left(\mathrm{C}^{\prime}-\mathrm{C}_{\infty}^{\prime}\right)
$$

The (9) becomes

$$
\begin{aligned}
& \rho\left(\frac{\partial \mathrm{u}^{\prime}}{\partial \mathrm{t}^{\prime}}+\mathrm{v}^{\prime} \frac{\partial \mathrm{u}^{\prime}}{\partial \mathrm{y}^{\prime}}\right)=\left(\rho_{\infty}-\rho\right) \mathrm{g}+\rho \frac{\mathrm{dU}_{\infty}^{\prime}}{\mathrm{dt}^{\prime}}+\mu \frac{\partial^{2} \mathrm{u}^{\prime}}{\partial \mathrm{y}^{\prime 2}}+\frac{\mu}{\mathrm{k}^{\prime}}\left(\mathrm{U}_{\infty}^{\prime}-\mathrm{u}^{\prime}\right)+\sigma \mathrm{B}_{0}^{2}\left(\mathrm{U}_{\infty}^{\prime}-\mathrm{u}^{\prime}\right) \\
& \frac{\partial \mathrm{u}^{\prime}}{\partial \mathrm{t}^{\prime}}+\mathrm{v}^{\prime} \frac{\partial \mathrm{u}^{\prime}}{\partial \mathrm{y}^{\prime}}=\frac{\mathrm{du}_{\infty}^{\prime}}{\mathrm{dt}^{\prime}}+\vartheta \frac{\partial^{2} \mathrm{u}^{\prime}}{\partial \mathrm{y}^{\prime 2}}+\mathrm{g} \beta\left(\mathrm{T}^{\prime}-\mathrm{T}_{\infty}^{\prime}\right)+\mathrm{g} \beta^{\prime}\left(\mathrm{C}^{\prime}-\mathrm{C}_{\infty}^{\prime}\right)+\frac{\mathrm{v}}{\mathrm{k}^{\prime}}\left(\mathrm{U}_{\infty}^{\prime}-\mathrm{u}^{\prime}\right)+ \\
& \frac{\sigma \mathrm{B}_{0}^{2}}{\rho}\left(\mathrm{U}_{\infty}^{\prime}-\mathrm{u}^{\prime}\right)
\end{aligned}
$$

The radioactive heat flux term by using the Roseland approximation is given by

$$
q_{r}^{\prime}=-\frac{4 \sigma^{\prime}}{3 k_{1}^{\prime}}{\frac{\partial T^{\prime}}{\partial y^{\prime}}}^{4}
$$

$\mathrm{T}^{\prime 4}$ may be expressed as a linear combination of the temperature.

This is accomplished by expanding in a Tayler series about $\mathrm{T}_{\infty}^{\prime}$ and neglecting higher order terms.

$$
\mathrm{T}^{\prime 4}=4 \mathrm{~T}^{\prime} \mathrm{T}_{\infty}^{\prime 4}-3 \mathrm{~T}_{\infty}^{\prime 4}
$$

by using (12) and (13)into (3) becomes

$$
\frac{\partial \mathrm{T}^{\prime}}{\partial \mathrm{t}^{\prime}}+\mathrm{v}^{\prime} \frac{\partial \mathrm{T}^{\prime}}{\partial \mathrm{y}^{\prime}}=\frac{\mathrm{k}}{\rho \mathrm{C}_{\mathrm{p}}} \frac{\partial^{2} \mathrm{~T}^{\prime}}{\partial \mathrm{y}^{\prime 2}}+\frac{16 \sigma^{\prime} \mathrm{T}_{\infty}^{\prime 3}}{3 \rho \mathrm{c}_{\mathrm{p}} \mathrm{k}_{1}^{\prime}} \frac{\partial^{2} \mathrm{~T}{ }^{\prime}}{\partial \mathrm{y}^{\prime^{2}}}-\frac{\mathrm{Q}_{0}}{\rho \mathrm{C}_{\mathrm{p}}}\left(\mathrm{T}^{\prime}-\mathrm{T}_{\infty}^{\prime}\right)+\frac{\mu}{\rho \mathrm{C}_{\mathrm{p}}}\left(\frac{\partial \mathrm{u}^{\prime}}{\partial \mathrm{y}^{\prime}}\right)^{2}
$$

To get the solution of (1) to (4) with boundary conditions (5) and (6), the following non dimensional parameters are used.

$$
\begin{gathered}
u=u U_{0}, v^{\prime}=v V_{0}, T^{\prime}=T_{\infty}^{\prime}+\theta\left(T_{w}^{\prime}-T_{\infty}^{\prime}\right), C^{\prime}=C_{\infty}^{\prime}+\varphi\left(C_{w}^{\prime}-C_{\infty}^{\prime}\right), U_{\infty}^{\prime}=U_{\infty} U_{0}, \\
u_{p}^{\prime}=U_{p} U_{0}, K^{\prime}=\frac{K v^{2}}{V_{0}{ }^{2}}, y^{\prime}=\frac{y v}{V_{0}}, G c=\frac{v g \beta^{\prime}\left(C_{w}^{\prime}-C_{\infty}^{\prime}\right)}{V_{0}{ }^{2} U_{0}}, G r=\frac{v g \beta\left(T_{w}^{\prime}-T_{\infty}^{\prime}\right)}{V_{0}{ }^{2} U_{0}}, R c=\frac{R c^{\prime} v}{V_{0}{ }^{2}}, \\
\operatorname{Pr}=\frac{v \rho C_{p}}{K}, M=\frac{\sigma B_{0}^{2}}{\rho V_{0}{ }^{2}}, Q=\frac{Q_{0} v}{\rho V_{0}{ }^{2} C_{p}}, R=\frac{4 \sigma^{\prime T_{\infty}^{\prime 3}}}{K_{1}^{\prime} K}, S c=\frac{v}{D_{M}}, t^{\prime}=\frac{t v}{V_{0}{ }^{2}}, n^{\prime}=\frac{n V_{0}{ }^{2}}{v}, S o=\frac{D_{T}\left(T_{w}^{\prime}-T_{\infty}^{\prime}\right)}{v\left(C_{w}^{\prime}-C_{\infty}^{\prime}\right)} \\
\mathrm{Ec}=\frac{U_{0}^{2}}{C_{p}\left(T_{w}^{\prime}-T_{\infty}^{\prime}\right)}
\end{gathered}
$$

Using the dimensionless parameters in (11), (14), and (4), we get

$$
\begin{aligned}
& \frac{\partial u}{\partial t}+v \frac{\partial u}{\partial y}=\frac{\partial^{2} u}{\partial y^{2}}+G r \theta+G c \varphi+N\left(U_{\infty}-U\right), \\
& \frac{\partial \theta}{\partial t}+v \frac{\partial \theta}{\partial y}=\frac{1}{P r}\left(1+\frac{4}{3} R\right) \frac{\partial^{2} \theta}{\partial y^{2}}-Q \theta+\operatorname{Ec}\left(\frac{\partial u}{\partial y}\right)^{2}, \\
& \frac{\partial \varphi}{\partial t}+v \frac{\partial \varphi}{\partial y}=\frac{1}{S c} \frac{\partial^{2} \varphi}{\partial y^{2}}+S o \frac{\partial^{2} \theta}{\partial y^{2}}-R c \varphi .
\end{aligned}
$$


$u=U_{p}, \theta=1+\epsilon e^{n t}, \theta \rightarrow 0, \varphi=1+\epsilon e^{n t}$ at $y=0$

$u \rightarrow U_{\infty}=1+\epsilon e^{n t}, \theta \rightarrow 0, \varphi \rightarrow 0$ as $y=\infty$

Perturbation method is used to solve (16), (17) and (18). The following forms are considered.

$$
\begin{aligned}
& u(y, t)=u_{0}(y)+\epsilon e^{n t} u_{1}(y)+O\left(\epsilon^{2}\right) \\
& \theta(y, t)=\theta_{0}(y)+\epsilon e^{n t} \theta_{1}(y)+O\left(\epsilon^{2}\right) \\
& \varphi(y, t)=\varphi_{0}(y)+\epsilon e^{n t} \varphi_{1}(y)+O\left(\epsilon^{2}\right)
\end{aligned}
$$

From (16), (17), and (18), we obtained

$$
\begin{aligned}
& u_{0}^{\prime \prime}+u_{0}^{\prime}-N u_{0}=-N-G r \theta_{0}-G c \varphi_{0}, \\
& u_{1}^{\prime \prime}+u_{1}^{\prime}-(N+n) u_{1}=-N-A u_{0}^{\prime}-\operatorname{Gr} \theta_{1}-G c \varphi_{1}, \\
& (3+4 R) \theta_{0}^{\prime \prime}+3 \operatorname{Pr} \theta_{0}^{\prime}-3 \operatorname{Pr} Q \theta_{0}=-3 \operatorname{Pr} E c u_{0}^{\prime 2}, \\
& (3+4 R) \theta_{1}^{\prime \prime}+3 \operatorname{Pr} \theta_{1}^{\prime}-3(n+Q) \operatorname{Pr} \theta_{1}=-3 \operatorname{APr} \theta_{0}^{\prime}-6 E c \operatorname{Pr} u_{0}^{\prime} u_{1}^{\prime}, \\
& \varphi_{0}^{\prime \prime}+\operatorname{Sc} \varphi_{0}^{\prime}-\operatorname{RcSc} \varphi_{0}=-\operatorname{SoS} c \theta_{0}^{\prime \prime}, \\
& \varphi_{1}^{\prime \prime}+\operatorname{Sc} \varphi_{1}^{\prime}-(R c+n) \operatorname{Sc} \varphi_{1}=-\operatorname{SoS} \operatorname{Sc} \theta_{1}^{\prime \prime}-\operatorname{ASc} \varphi_{0}^{\prime},
\end{aligned}
$$

Corresponding boundary conditions are

$$
\begin{array}{lr}
u_{00}=U_{p}, u_{01}=0, \theta_{00}=1, \theta_{01}=1, \varphi_{00}=1, \varphi_{01}=1 & \text { as } \mathrm{y}=0 \\
u_{10}=U_{p}, u_{11}=0, \theta_{10}=1, \theta_{11}=1, \varphi_{10}=1, \varphi_{11}=1 & \\
u_{00} \rightarrow 1, u_{01} \rightarrow 1, \theta_{00} \rightarrow 0, \theta_{01} \rightarrow 0, \varphi_{00} \rightarrow 0, \varphi_{01} \rightarrow 0 & \text { as } \mathrm{y} \rightarrow \infty \\
u_{10} \rightarrow 1, u_{11} \rightarrow 1, \theta_{10} \rightarrow 0, \theta_{11} \rightarrow 0, \varphi_{10} \rightarrow 0, \varphi_{11} \rightarrow 0 &
\end{array}
$$

Second Perturbation technique is used to solve (20), (21), (22), (23), (24), and (25). The following forms are considered

$$
\begin{aligned}
& u_{0}(y, t)=u_{00}(y)+E c u_{01}(y)+O\left(E c^{2}\right) \\
& u_{1}(y, t)=u_{10}(y)+E c u_{11}(y)+O\left(E c^{2}\right) \\
& \theta_{0}(y, t)=\theta_{00}(y)+E c \theta_{01}(y)+O\left(E c^{2}\right) \\
& \theta_{1}(y, t)=\theta_{10}(y)+E c \theta_{11}(y)+O\left(E c^{2}\right) \\
& \varphi_{0}(y, t)=\varphi_{00}(y)+E c \varphi_{01}(y)+O\left(E c^{2}\right) \\
& \varphi_{1}(y, t)=\varphi_{10}(y)+E c \varphi_{11}(y)+O\left(E c^{2}\right)
\end{aligned}
$$

From (20), (21),(22),(23),(24) and (25), we obtained

$$
\begin{aligned}
& u_{00}^{\prime \prime}+u_{00}^{\prime}-N u_{00}=-N-G r \theta_{00}-G c \varphi_{00} \\
& u_{01}^{\prime \prime}+u_{01}^{\prime}-N u_{01}=-N-G r \theta_{01}-G c \varphi_{01}, \\
& u_{10}^{\prime \prime}+u_{10}^{\prime}-(N+n) u_{10}=-N-A u_{00}^{\prime}-G r \theta_{10}-G c \varphi_{10}, \\
& u_{11}^{\prime \prime}+u_{11}^{\prime}-(N+n) u_{11}=-N-A u_{01}^{\prime}-G r \theta_{11}-G c \varphi_{11} \\
& (3+4 R) \theta_{00}^{\prime \prime}+3 \operatorname{Pr} \theta_{00}^{\prime}-3 \operatorname{Pr} Q \theta_{00}=0 \\
& (3+4 R) \theta_{01}^{\prime \prime}+3 \operatorname{Pr} \theta_{01}^{\prime}-3 \operatorname{Pr} Q \theta_{01}=-3 \operatorname{Pr} u_{00}^{\prime 2} \\
& (3+4 R) \theta_{10}^{\prime \prime}+3 \operatorname{Pr} \theta_{10}^{\prime}-3 \operatorname{Pr}(Q+n) \theta_{10}=-3 A \operatorname{Pr} \theta_{00}^{\prime}
\end{aligned}
$$




$$
\begin{aligned}
& (3+4 R) \theta_{11}^{\prime \prime}+3 \operatorname{Pr} \theta_{11}^{\prime}-3(Q+n) \operatorname{Pr} \theta_{11}=-3 \operatorname{APr} \theta_{01}^{\prime}-6 \operatorname{Pr} u_{00}^{\prime} u_{10}^{\prime} \\
& \varphi_{00}^{\prime \prime}+S c \varphi_{00}^{\prime}-R c S c \varphi_{00}=-\operatorname{SoSc} \theta_{00}^{\prime \prime} \\
& \varphi_{01}^{\prime \prime}+S c \varphi_{01}^{\prime}-R c S c \varphi_{01}=-\operatorname{SoSc} \theta_{01}^{\prime \prime} \\
& \varphi_{10}^{\prime \prime}+S c \varphi_{10}^{\prime}-(R c+n) S c \varphi_{10}=-\operatorname{SoSc} \theta_{10}^{\prime \prime}-A S c \varphi_{00} \\
& \varphi_{11}^{\prime \prime}+\operatorname{Sc} \varphi_{11}^{\prime}-(R c+n) S c \varphi_{11}=-\operatorname{SoSc} \theta_{11}^{\prime \prime}-\operatorname{SSc} \varphi_{01}
\end{aligned}
$$

Solving (31) and (33), we obtained

$$
\begin{aligned}
& \theta_{00}=e^{m_{2} y}, \\
& \theta_{10}=C_{1} e^{m_{2} y}+C_{2} e^{m_{4} y},
\end{aligned}
$$

Solving (35) and (37), we obtained

$$
\begin{aligned}
& \varphi_{00}=B_{1} e^{m_{2} y}+B_{2} e^{m_{6} y} \\
& \varphi_{10}=B_{3} e^{m_{6} y}+B_{4} e^{m_{2} y}+B_{5} e^{m_{8} y}+C_{3} e^{m_{2} y}+C_{4} e^{m_{4} y}
\end{aligned}
$$

Solving (27) and (32), we obtained

$$
\begin{aligned}
& u_{00}=1+A_{1} e^{m_{2} y}+A_{2} e^{m_{6} y}+A_{3} e^{m_{2} y}+A_{4} e^{m_{10} y} \\
& \theta_{01}=E_{1} e^{2 m_{2} y}+E_{2} e^{2 m_{6} y}+E_{3} e^{2 m_{2} y}+E_{4} e^{2 m_{10} y}+E_{5} e^{\left(m_{2}+m_{6}\right) y}+E_{6} e^{\left(m_{2}+m_{10}\right) y}+ \\
& E_{7} e^{2 m_{2} y}+E_{8} e^{\left(m_{2}+m_{10}\right) y}+E_{9} e^{\left(m_{2}+m_{6}\right) y}+E_{10} e^{\left(m_{6}+m_{10}\right) y}+E_{11} e^{m_{12} y}
\end{aligned}
$$

Solving (36) and (28), we obtained

$$
\begin{aligned}
& \varphi_{01}=C_{5} e^{2 m_{2} y}+C_{6} e^{2 m_{6} y}+C_{7} e^{\left(m_{2}+m_{6}\right) y}+C_{8} e^{2 m_{2} y}+C_{9} e^{2 m_{10} y}+C_{10} e^{\left(m_{2}+m_{6}\right) y}+ \\
& C_{11} e^{2 m_{2} y}+C_{12} e^{\left(m_{2}+m_{10}\right) y}+C_{13} e^{\left(m_{2}+m_{6}\right) y}+C_{14} e^{\left(m_{10}+m_{6}\right) y}+C_{15} e^{m_{12} y}+C_{16} e^{m_{12} y}(45) \\
& u_{01}=1+A_{18} e^{2 m_{2} y}+A_{19} e^{2 m_{6} y}+A_{20} e^{\left(m_{2}+m_{6}\right) y}+A_{21} e^{2 m_{2} y}+A_{22} e^{2 m_{10} y}+ \\
& A_{23} e^{\left(m_{2}+m_{6}\right) y}+A_{24} e^{2 m_{2} y}+A_{25} e^{\left(m_{2}+m_{10}\right) y}+A_{26} e^{\left(m_{2}+m_{6}\right) y}+A_{27} e^{\left(m_{10}+m_{6}\right) y}+ \\
& A_{28} e^{m_{12} y}+A_{29} e^{2 m_{2} y}+A_{30} e^{2 m_{6} y}+A_{31} e^{\left(m_{2}+m_{6}\right) y}+A_{32} e^{2 m_{2} y}+A_{33} e^{2 m_{2} y}+ \\
& A_{34} e^{\left(m_{2}+m_{6}\right) y}+A_{35} e^{2 m_{2} y}+A_{36} e^{\left(m_{2}+m_{6}\right) y}+A_{37} e^{\left(m_{2}+m_{6}\right) y}+A_{38} e^{\left(m_{10}+m_{6}\right) y}+ \\
& A_{39} e^{m_{12} y}+A_{40} e^{m_{14} y+A_{41} e^{m_{16} y}} .
\end{aligned}
$$

Solving (29) and (34), we obtained

$$
\begin{aligned}
& u_{10}=1+A_{42} e^{m_{2} y}+A_{43} e^{m_{8} y}+A_{44} e^{m_{2} y}+A_{45} e^{m_{10} y}+A_{46} e^{m_{2} y}+A_{47} e^{m_{4} y}+ \\
& A_{48} e^{m_{8} y}+A_{49} e^{m_{2} y}+A_{50} e^{m_{2} y}+A_{51} e^{m_{6} y}+A_{52} e^{m_{18} y} \\
& \theta_{11}=A_{52} e^{2 m_{2} y}+A_{53} e^{2 m_{6} y}+A_{54} e^{\left(m_{2}+m_{6}\right) y}+A_{55} e^{2 m_{4} y}+A_{56} e^{\left(m_{2}+m_{6}\right) y}+A_{57} e^{2 m_{2} y}+ \\
& A_{58} e^{\left(m_{2}+m_{6}\right) y}+A_{59} e^{\left(m_{2}+m_{6}\right) y}+A_{60} e^{\left(m_{10}+m_{6}\right) y}+A_{61} e^{\left(m_{10}+m_{6}\right) y}+A_{62} e^{m_{20} y}+ \\
& E_{11} e^{2 m_{2} y}+E_{12} e^{\left(m_{8}+m_{2}\right) y}+E_{13} e^{2 m_{2} y}+E_{14} e^{\left(m_{10}+m_{2}\right) y}+E_{15} e^{2 m_{2} y}+E_{16} e^{\left(m_{2}+m_{4}\right) y}+ \\
& E_{17} e^{\left(m_{8}+m_{2}\right) y}+E_{18} e^{2 m_{2} y}+E_{19} e^{2 m_{2} y}+E_{20} e^{\left(m_{2}+m_{6}\right) y}+E_{21} e^{\left(m_{8}+m_{2}\right) y}+E_{22} e^{\left(m_{2}+m_{6}\right) y}+ \\
& E_{23} e^{\left(m_{2}+m_{6}\right) y}+E_{24} e^{\left(m_{2}+m_{6}\right) y}+E_{25} e^{\left(m_{6}+m_{10}\right) y}+E_{26} e^{\left(m_{2}+m_{6}\right) y}+E_{27} e^{\left(m_{4}+m_{6}\right) y}+ \\
& E_{28} e^{\left(m_{8}+m_{6}\right) y}+E_{29} e^{\left(m_{2}+m_{6}\right) y}+E_{30} e^{\left(m_{2}+m_{6}\right) y}+E_{31} e^{2 m_{2} y}+E_{32} e^{\left(m_{2}+m_{8}\right) y}+E_{33} e^{2 m_{2} y}+ \\
& E_{34} e^{\left(m_{2}+m_{8}\right) y}+E_{35} e^{2 m_{2} y}+E_{36} e^{\left(m_{10}+m_{2}\right) y}+E_{37} e^{2 m_{2} y}+E_{38} e^{\left(m_{2}+m_{4}\right) y}+ \\
& E_{39} e^{\left(m_{2}+m_{8}\right) y}+E_{40} e^{2 m_{2} y}+E_{41} e^{2 m_{2} y}+E_{42} e^{\left(m_{2}+m_{6}\right) y}+E_{43} e^{\left(m_{2}+m_{18}\right) y}+ \\
& E_{44} e^{\left(m_{2}+m_{10}\right) y}+E_{45} e^{\left(m_{8}+m_{10}\right) y}+E_{46} e^{\left(m_{10}+m_{2}\right) y}+E_{47} e^{2 m_{10} y}+E_{48} e^{\left(m_{10}+m_{2}\right) y}+ \\
& E_{49} e^{\left(m_{10}+m_{4}\right) y}+E_{50} e^{\left(m_{8}+m_{10}\right) y}+E_{51} e^{\left(m_{10}+m_{2}\right) y}+E_{52} e^{\left(m_{10}+m_{2}\right) y}+E_{53} e^{\left(m_{10}+m_{6}\right) y}+ \\
& E_{54} e^{\left(m_{10}+m_{18}\right) y} .
\end{aligned}
$$


Solving (30) and (38), we obtained

$$
\begin{aligned}
& \varphi_{11}=C_{17} e^{2 m_{2} y}+C_{18} e^{2 m_{6} y}+C_{19} e^{\left(m_{2}+m_{8}\right) y}+C_{20} e^{2 m_{4} y}+C_{21} e^{\left(m_{2}+m_{6}\right) y}+C_{22} e^{2 m_{2} y}+ \\
& C_{23} e^{\left(m_{2}+m_{10}\right) y}+C_{24} e^{\left(m_{2}+m_{6}\right) y}+C_{25} e^{\left(m_{6}+m_{10}\right) y}+C_{26} e^{m_{12} y}+C_{27} e^{m_{20} y}+C_{28} e^{2 m^{2} y}+ \\
& C_{29} e^{\left(m_{2}+m_{6}\right) y}+C_{30} e^{2 m_{2} y}+C_{31} e^{\left(m_{2}+m_{10}\right) y}+C_{32} e^{2 m_{2} y}+C_{33} e^{\left(m_{2}+m_{4}\right) y}+ \\
& C_{34} e^{\left(m_{2}+m_{8}\right) y}+C_{35} e^{2 m_{2} y}+C_{36} e^{2 m_{2} y}+C_{37} e^{\left(m_{2}+m_{6}\right) y}+C_{38} e^{\left(m_{2}+m_{8}\right) y}+C_{39} e^{\left(m_{2}+m_{8}\right) y}+ \\
& C_{40} e^{\left(m_{2}+m_{6}\right) y}+C_{41} e^{\left(m_{2}+m_{6}\right) y}+C_{42} e^{\left(m_{6}+m_{10}\right) y}+C_{43} e^{\left(m_{2}+m_{6}\right) y}+C_{44} e^{\left(m_{4}+m_{6}\right) y}+ \\
& C_{45} e^{\left(m_{6}+m_{8}\right) y}+C_{46} e^{\left(m_{2}+m_{6}\right) y}+C_{47} e^{\left(m_{2}+m_{6}\right) y}+C_{48} e^{2 m_{6} y}+C_{49 e^{\left(m_{2}+m_{8}\right) y}}+C_{50} e^{2 m_{2} y}+ \\
& C_{51} e^{\left(m_{2}+m_{8}\right) y}+C_{52} e^{2 m_{2} y}+C_{53} e^{\left(m_{2}+m_{10}\right) y}+C_{54} e^{2 m_{2} y}+C_{55} e^{\left(m_{2}+m_{4}\right) y}+ \\
& C_{56} e^{\left(m_{2}+m_{8}\right) y}+C_{57} e^{2 m_{2} y}+C_{58} e^{2 m_{2} y}+C_{59} e^{\left(m_{2}+m_{6}\right) y}+C_{60} e^{\left(m_{2}+m_{18}\right) y}+ \\
& C_{61} e^{\left(m_{2}+m_{10}\right) y}+C_{62} e^{\left(m_{8}+m_{10}\right) y}+C_{63} e^{\left(m_{2}+m_{10}\right) y}+C_{64} e^{2 m_{10} y}+C_{65} e^{\left(m_{2}+m_{10}\right) y}+C_{66} \\
& e^{\left(m_{4}+m_{10}\right) y}+C_{67} e^{\left(m_{8}+m_{10}\right) y}+C_{68} e^{\left(m_{2}+m_{10}\right) y}+C_{69} e^{\left(m_{2}+m_{10}\right) y}+C_{70} e^{\left(m_{6}+m_{10}\right) y}+ \\
& C_{71} e^{\left(m_{10}+m_{18}\right) y}+C_{72} e^{2 m_{2} y}+C_{73} e^{2 m_{6} y}+C_{74} e^{\left(m_{2}+m_{6}\right) y}+C_{75} e^{2 m_{2} y}+C_{76} e^{2 m_{10} y}+C_{77} \\
& e^{\left(m_{2}+m_{6}\right) y}+C_{78} e^{2 m_{2} y}+C_{79} e^{\left(m_{2}+m_{10}\right) y_{1}}+C_{80} e^{\left(m_{2}+m_{6}\right) y}+C_{81} e^{\left(m_{6}+m_{10}\right) y}+C_{82} e^{m_{2} y}+ \\
& C_{83} e^{m_{14} y}+C_{84} e^{m_{22} y} \text {, } \\
& u_{11}=1+C_{85} e^{2 m_{2} y}+C_{86} e^{2 m_{6} y}+C_{87} e^{\left(m_{2}+m_{6}\right) y}+C_{88} e^{2 m_{2} y}+C_{89} e^{2 m_{10} y}+ \\
& C_{90} e^{\left(m_{2}+m_{10}\right) y}+C_{91} e^{2 m_{2} y}+C_{92} e^{\left(m_{2}+m_{10}\right) y}+C_{93} e^{\left(m_{2}+m_{6}\right) y}+C_{94} e^{\left(m_{6}+m_{10}\right) y}+ \\
& C_{95} e^{2 m_{12} y}+C_{96} e^{2 m_{2} y}+C_{97} e^{2 m_{6} y}+C_{98} e^{\left(m_{2}+m_{6}\right) y}+C_{99} e^{2 m_{2} y}+C_{100} e^{2 m_{2} y}+ \\
& C_{101} e^{\left(m_{2}+m_{6}\right) y}+C_{102} e^{2 m_{2} y}+C_{103} e^{\left(m_{2}+m_{6}\right) y}+C_{104} e^{\left(m_{2}+m_{6}\right) y}+C_{105} e^{\left(m_{6}+m_{10}\right) y}+ \\
& C_{106} e^{m_{12} y}+C_{107} e^{m_{14} y}+C_{108} e^{m_{16} y}+C_{109} e^{2 m_{2} y}+C_{110} e^{2 m_{6} y}+C_{111} e^{\left(m_{2}+m_{6}\right) y}+C_{112} \\
& e^{2 m_{4} y}+C_{113} e^{\left(m_{2}+m_{6}\right) y}+C_{114} e^{2 m_{2} y}+C_{115} e^{\left(m_{2}+m_{10}\right) y}+C_{116} e^{\left(m_{2}+m_{6}\right) y}+ \\
& C_{117} e^{\left(m_{6}+m_{10}\right) y}+C_{118} e^{m_{12} y}+C_{119} e^{m_{20} y}+C_{120} e^{2 m_{2} y}+C_{121} e^{\left(m_{2}+m_{6}\right) y}+C_{122} e^{2 m_{2} y}+ \\
& C_{123} e^{\left(m_{2}+m_{10}\right) y}+C_{124} e^{2 m_{2} y}+C_{125} e^{\left(m_{2}+m_{4}\right) y}+C_{126} e^{\left(m_{2}+m_{18}\right) y}+C_{127} e^{2 m_{2} y}+ \\
& C_{128} e^{2 m_{2} y}+C_{129} e^{\left(m_{2}+m_{6}\right) y}+C_{130} e^{\left(m_{2}+m_{8}\right) y}+C_{131} e^{\left(m_{2}+m_{6}\right) y}+C_{132} e^{\left(m_{2}+m_{6}\right) y}+ \\
& C_{133} e^{\left(m_{2}+m_{6}\right) y}+C_{134} e^{\left(m_{10}+m_{6}\right) y}+C_{135} e^{\left(m_{2}+m_{6}\right) y}+C_{136} e^{\left(m_{4}+m_{6}\right) y}+C_{137} e^{\left(m_{8}+m_{6}\right) y}+ \\
& C_{138} e^{\left(m_{2}+m_{6}\right) y}+C_{139} e^{\left(m_{2}+m_{6}\right) y}+C_{140} e^{2 m_{6} y}+C_{141} e^{\left(m_{2}+m_{8}\right) y}+C_{142} e^{2 m_{2} y}+ \\
& C_{143} e^{\left(m_{2}+m_{8}\right) y}+C_{144} e^{2 m_{2} y}+C_{145} e^{\left(m_{2}+m_{10}\right) y}+C_{146} e^{2 m_{2} y}+C_{147} e^{\left(m_{2}+m_{4}\right) y}+ \\
& C_{148} e^{\left(m_{2}+m_{8}\right) y}+C_{149} e^{2 m_{2} y}+C_{150} e^{2 m_{2} y}+C_{151} e^{\left(m_{2}+m_{6}\right) y}+C_{152} e^{\left(m_{2}+m_{18}\right) y}+ \\
& C_{153} e^{\left(m_{2}+m_{10}\right) y}+C_{154} e^{\left(m_{8}+m_{10}\right) y}+C_{155} e^{\left(m_{2}+m_{10}\right) y}+C_{156} e^{2 m_{10} y}+C_{157} e^{\left(m_{2}+m_{10}\right) y}+ \\
& C_{158} e^{\left(m_{4}+m_{10}\right) y}+C_{159} e^{\left(m_{8}+m_{10}\right) y}+C_{160} e^{\left(m_{2}+m_{10}\right) y}+C_{161} e^{\left(m_{2}+m_{10}\right) y}+C_{162} e^{\left(m_{6}+m_{10}\right) y}+ \\
& C_{163} e^{\left(m_{18}+m_{10}\right) y}+C_{164} e^{2 m_{2} y}+C_{165} e^{2 m_{6} y}+C_{166} e^{\left(m_{2}+m_{6}\right) y}+C_{167} e^{2 m_{4} y}+ \\
& C_{168} e^{\left(m_{2}+m_{6}\right) y}+C_{169} e^{2 m_{2} y}+C_{170} e^{\left(m_{2}+m_{10}\right) y}+C_{171} e^{\left(m_{2}+m_{6}\right) y}+C_{172} e^{\left(m_{6}+m_{10}\right) y}+ \\
& C_{173} e^{m_{12} y}+C_{174} e^{m_{20} y}+C_{175} e^{2 m_{2} y}+C_{176} e^{\left(m_{2}+m_{6}\right) y}+C_{177} e^{2 m_{2} y}+C_{178} e^{\left(m_{2}+m_{10}\right) y}+ \\
& C_{179} e^{2 m_{2} y}+C_{180} e^{\left(m_{2}+m_{4}\right) y}+C_{181} e^{\left(m_{2}+m_{8}\right) y}+C_{182} e^{2 m_{2} y}+C_{183} e^{2 m_{2} y}+C_{184} \\
& e^{\left(m_{2}+m_{6}\right) y}+C_{185} e^{\left(m_{2}+m_{8}\right) y}+C_{186} e^{\left(m_{2}+m_{6}\right) y}+C_{187} e^{\left(m_{2}+m_{6}\right) y}+C_{188} e^{\left(m_{2}+m_{6}\right) y}+ \\
& C_{189} e^{\left(m_{10}+m_{6}\right) y}+C_{190} e^{\left(m_{2}+m_{6}\right) y}+C_{191} e^{\left(m_{4}+m_{6}\right) y}+C_{192} e^{\left(m_{8}+m_{6}\right) y}+C_{193} e^{\left(m_{2}+m_{6}\right) y}+ \\
& C_{194 e\left(m_{2}+m_{6}\right) y}+C_{195} e^{2 m_{6} y}+C_{196} e^{\left(m_{8}+m_{2}\right) y}+C_{197} e^{2 m_{2} y}+C_{198} e^{\left(m_{8}+m_{2}\right) y}+C_{199} e^{2 m_{2} y}+ \\
& C_{200} e^{\left(m_{10}+m_{2}\right) y}+C_{201} e^{2 m_{2} y}+C_{202} e^{\left(m_{4}+m_{2}\right) y}+C_{203} e^{\left(m_{8}+m_{2}\right) y}+C_{204} e^{2 m_{2} y}+ \\
& C_{205} e^{2 m_{2} y}+C_{206} e^{\left(m_{2}+m_{6}\right) y}+C_{207} e^{\left(m_{2}+m_{18}\right) y}+C_{208} e^{\left(m_{10}+m_{2}\right) y}+C_{209} e^{\left(m_{10}+m_{8}\right) y}+ \\
& C_{210} e^{\left(m_{10}+m_{2}\right) y}+C_{211} e^{2 m_{10} y}+C_{212} e^{\left(m_{10}+m_{2}\right) y}+C_{213} e^{\left(m_{10}+m_{4}\right) y}+C_{214} e^{\left(m_{10}+m_{8}\right) y}+ \\
& C_{215} e^{\left(m_{10}+m_{2}\right) y}+C_{216} e^{\left(m_{10}+m_{2}\right) y}+C_{217} e^{\left(m_{10}+m_{6}\right) y}+C_{218} e^{\left(m_{10}+m_{18}\right) y}+C_{219} e^{2 m_{2} y}+ \\
& C_{220} e^{2 m_{6} y}+C_{221} e^{\left(m_{2}+m_{6}\right) y}+C_{222} e^{2 m_{2} y}+C_{223} e^{2 m_{10} y}+C_{224} e^{\left(m_{2}+m_{6}\right) y}+C_{225} e^{2 m_{2} y}+ \\
& C_{226} e^{\left(m_{2}+m_{10}\right) y}+C_{227} e^{\left(m_{2}+m_{6}\right) y}+C_{228} e^{\left(m_{10}+m_{6}\right) y}+C_{229} e^{m_{12} y}+C_{230} e^{m_{14} y}+ \\
& C_{231} e^{m_{22} y}+C_{232} e^{2 m_{2} y}+C_{233} e^{m_{24} y}
\end{aligned}
$$




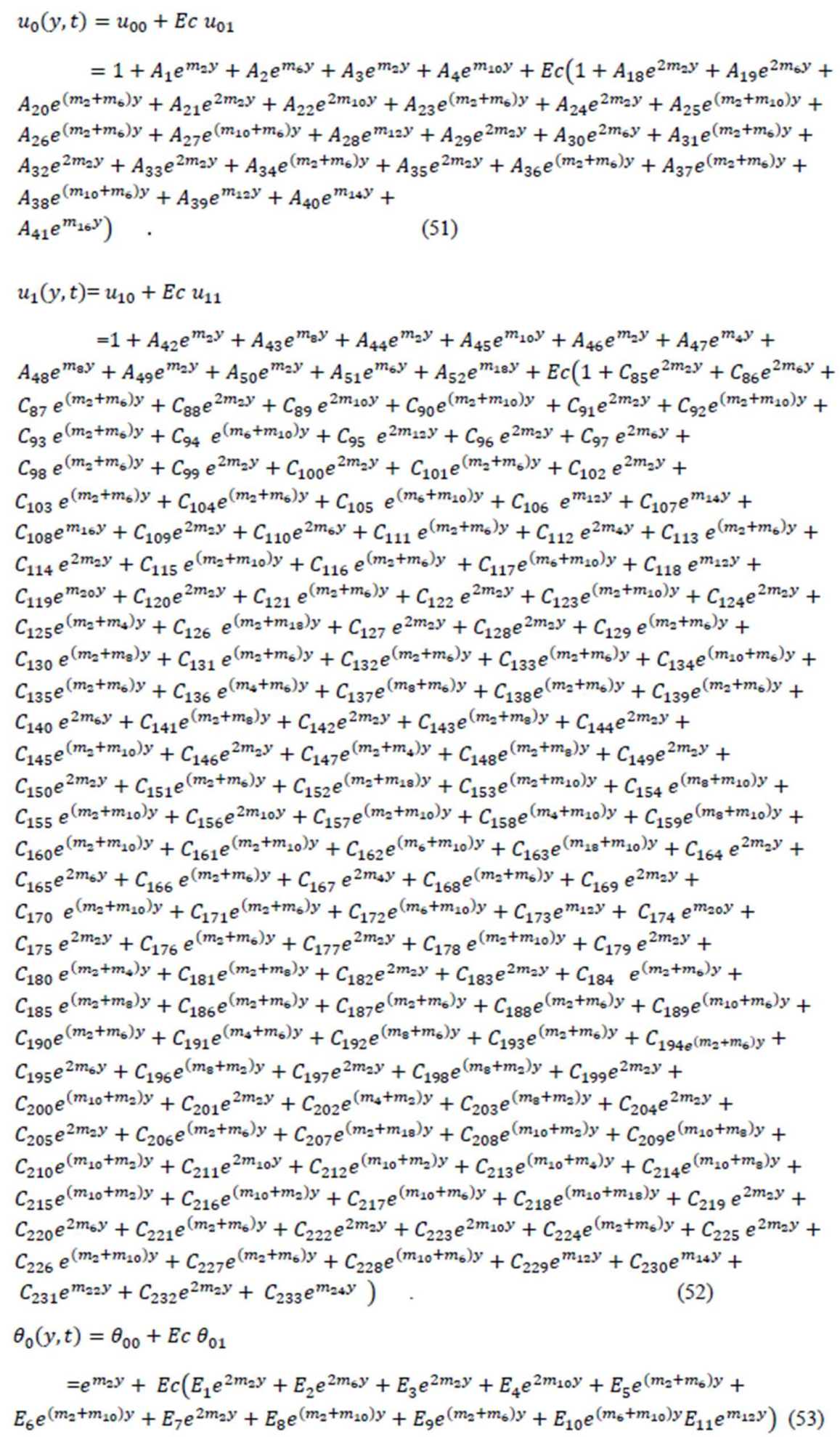

$u_{1}(y, t)=u_{10}+E c u_{11}$

$=1+A_{42} e^{m_{2} y}+A_{43} e^{m_{8} y}+A_{44} e^{m_{2} y}+A_{45} e^{m_{10} y}+A_{46} e^{m_{2} y}+A_{47} e^{m_{4} y}+$

$A_{48} e^{m_{8} y}+A_{49} e^{m_{2} y}+A_{50} e^{m_{2} y}+A_{51} e^{m_{6} y}+A_{52} e^{m_{18} y}+E c\left(1+C_{85} e^{2 m_{2} y}+C_{86} e^{2 m_{6} y}+\right.$ $C_{87} e^{\left(m_{2}+m_{6}\right) y}+C_{88} e^{2 m_{2} y}+C_{89} e^{2 m_{10} y}+C_{90} e^{\left(m_{2}+m_{10}\right) y}+C_{91} e^{2 m_{2} y}+C_{92} e^{\left(m_{2}+m_{10}\right) y}+$ 


$$
\begin{aligned}
& \theta_{1}(y, t)=\theta_{10}+E c \theta_{11} \\
& =C_{1} e^{m_{2} y}+C_{2} e^{m_{4} y}+E c\left(A_{52} e^{2 m_{2} y}+A_{53} e^{2 m_{6} y}+A_{54} e^{\left(m_{2}+m_{6}\right) y}+A_{55} e^{2 m_{4} y}+\right. \\
& A_{56} e^{\left(m_{2}+m_{6}\right) y}+A_{57} e^{2 m_{2} y}+A_{58} e^{\left(m_{2}+m_{6}\right) y}+A_{59} e^{\left(m_{2}+m_{6}\right) y}+A_{60} e^{\left(m_{10}+m_{6}\right) y}+ \\
& A_{61} e^{\left(m_{10}+m_{6}\right) y}+A_{62} e^{m_{20} y}+E_{11} e^{2 m_{2} y}+E_{12} e^{\left(m_{8}+m_{2}\right) y}+E_{13} e^{2 m_{2} y}+E_{14} e^{\left(m_{10}+m_{2}\right) y}+ \\
& E_{15} e^{2 m_{2} y}+E_{16} e^{\left(m_{2}+m_{4}\right) y}+E_{17} e^{\left(m_{8}+m_{2}\right) y}+E_{18} e^{2 m_{2} y}+E_{19} e^{2 m_{2} y}+E_{20} e^{\left(m_{2}+m_{6}\right) y}+ \\
& E_{21} e^{\left(m_{\mathrm{s}}+m_{2}\right) y}+E_{22} e^{\left(m_{2}+m_{6}\right) y}+E_{23} e^{\left(m_{2}+m_{6}\right) y}+E_{24} e^{\left(m_{2}+m_{6}\right) y}+E_{25} e^{\left(m_{6}+m_{10}\right) y}+ \\
& E_{26} e^{\left(m_{2}+m_{6}\right) y}+E_{27} e^{\left(m_{4}+m_{6}\right) y}+E_{28} e^{\left(m_{8}+m_{6}\right) y}+E_{29} e^{\left(m_{2}+m_{6}\right) y}+E_{30} e^{\left(m_{2}+m_{6}\right) y}+ \\
& E_{31} e^{2 m_{2} y}+E_{32} e^{\left(m_{2}+m_{8}\right) y}+E_{33} e^{2 m_{2} y}+E_{34} e^{\left(m_{2}+m_{8}\right) y}+E_{35} e^{2 m_{2} y}+E_{36} e^{\left(m_{10}+m_{2}\right) y}+ \\
& E_{37} e^{2 m_{2} y}+E_{38} e^{\left(m_{2}+m_{4}\right) y}+E_{39} e^{\left(m_{2}+m_{8}\right) y}+E_{40} e^{2 m_{2} y}+E_{41} e^{2 m_{2} y}+E_{42} e^{\left(m_{2}+m_{6}\right) y}+ \\
& E_{43} e^{\left(m_{2}+m_{18}\right) y}+E_{44} e^{\left(m_{2}+m_{10}\right) y}+E_{45} e^{\left(m_{8}+m_{10}\right) y}+E_{46} e^{\left(m_{10}+m_{2}\right) y}+E_{47} e^{2 m_{10} y}+ \\
& E_{48} e^{\left(m_{10}+m_{2}\right) y}+E_{49} e^{\left(m_{10}+m_{4}\right) y}+E_{50} e^{\left(m_{8}+m_{10}\right) y}+E_{51} e^{\left(m_{10}+m_{2}\right) y}+E_{52} e^{\left(m_{10}+m_{2}\right) y}+ \\
& \left.E_{53} e^{\left(m_{10}+m_{6}\right) y}+E_{54} e^{\left(m_{10}+m_{18}\right) y}\right) \text {. } \\
& \varphi_{0}(y, t)=\varphi_{00}+E c \varphi_{01} \\
& =B_{1} e^{m_{2} y}+B_{2} e^{m_{6} y}+\mathrm{Ec}\left(C_{5} e^{2 m_{2} y}+C_{6} e^{2 m_{6} y}+C_{7} e^{\left(m_{2}+m_{6}\right) y}+C_{8} e^{2 m_{2} y}+\right. \\
& C_{9} e^{2 m_{10} y}+C_{10} e^{\left(m_{2}+m_{6}\right) y}+C_{11} e^{2 m_{2} y}+C_{12} e^{\left(m_{2}+m_{10}\right) y}+C_{13} e^{\left(m_{2}+m_{6}\right) y}+ \\
& \left.C_{14} e^{\left(m_{10}+m_{6}\right) y}+C_{15} e^{m_{12} y}+C_{16} e^{m_{12} y}\right) \\
& \varphi_{1}(y, t)=\varphi_{10}+E c \varphi_{11} \\
& =B_{3} e^{m_{6} y}+B_{4} e^{m_{2} y}+B_{5} e^{m_{8} y}+C_{3} e^{m_{2} y}+C_{4} e^{m_{4} y}+E c\left(C_{17} e^{2 m_{2} y}+\right. \\
& C_{18} e^{2 m_{6} y}+C_{19} e^{\left(m_{2}+m_{8}\right) y}+C_{20} e^{2 m_{4} y}+C_{21} e^{\left(m_{2}+m_{6}\right) y}+C_{22} e^{2 m_{2} y}+C_{23} e^{\left(m_{2}+m_{10}\right) y}+ \\
& C_{24} e^{\left(m_{2}+m_{6}\right) y}+C_{25} e^{\left(m_{6}+m_{10}\right) y}+C_{26} e^{m_{12} y}+C_{27} e^{m_{20} y}+C_{28} e^{2 m^{2} y}+C_{29} e^{\left(m_{2}+m_{6}\right) y}+ \\
& C_{30} e^{2 m_{2} y}+C_{31} e^{\left(m_{2}+m_{10}\right) y}+C_{32} e^{2 m_{2} y}+C_{33} e^{\left(m_{2}+m_{4}\right) y}+C_{34} e^{\left(m_{2}+m_{8}\right) y}+C_{35} e^{2 m_{2} y}+ \\
& C_{36} e^{2 m_{2} y}+C_{37} e^{\left(m_{2}+m_{6}\right) y}+C_{38} e^{\left(m_{2}+m_{8}\right) y}+C_{39} e^{\left(m_{2}+m_{8}\right) y}+C_{40} e^{\left(m_{2}+m_{6}\right) y}+ \\
& C_{41} e^{\left(m_{2}+m_{6}\right) y}+C_{42} e^{\left(m_{6}+m_{10}\right) y}+C_{43} e^{\left(m_{2}+m_{6}\right) y}+C_{44} e^{\left(m_{4}+m_{6}\right) y}+C_{45} e^{\left(m_{6}+m_{8}\right) y}+ \\
& C_{46} e^{\left(m_{2}+m_{6}\right) y}+C_{47} e^{\left(m_{2}+m_{6}\right) y}+C_{48} e^{2 m_{6} y}+C_{49 e}\left(m_{2}+m_{8}\right) y+C_{50} e^{2 m_{2} y}+C_{51} e^{\left(m_{2}+m_{8}\right) y}+ \\
& C_{52} e^{2 m_{2} y}+C_{53} e^{\left(m_{2}+m_{10}\right) y}+C_{54} e^{2 m_{2} y}+C_{55} e^{\left(m_{2}+m_{4}\right) y}+C_{56} e^{\left(m_{2}+m_{8}\right) y}+C_{57} e^{2 m_{2} y}+ \\
& C_{58} e^{2 m_{2} y}+C_{59} e^{\left(m_{2}+m_{6}\right) y}+C_{60} e^{\left(m_{2}+m_{18}\right) y}+C_{61} e^{\left(m_{2}+m_{10}\right) y}+C_{62} e^{\left(m_{8}+m_{10}\right) y}+ \\
& C_{63} e^{\left(m_{2}+m_{10}\right) y}+C_{64} e^{2 m_{10} y}+C_{65} e^{\left(m_{2}+m_{10}\right) y}+C_{66} e^{\left(m_{4}+m_{10}\right) y}+C_{67} e^{\left(m_{8}+m_{10}\right) y}+ \\
& C_{68} e^{\left(m_{2}+m_{10}\right) y}+C_{69} e^{\left(m_{2}+m_{10}\right) y}+C_{70} e^{\left(m_{6}+m_{10}\right) y}+C_{71} e^{\left(m_{10}+m_{18}\right) y}+C_{72} e^{2 m_{2} y}+ \\
& C_{73} e^{2 m_{6} y}+C_{74} e^{\left(m_{2}+m_{6}\right) y}+C_{75} e^{2 m_{2} y}+C_{76} e^{2 m_{10} y}+C_{77} e^{\left(m_{2}+m_{6}\right) y}+C_{78} e^{2 m_{2} y}+ \\
& C_{79} e^{\left(m_{2}+m_{10}\right) y}+C_{80} e^{\left(m_{2}+m_{6}\right) y}+C_{81} e^{\left(m_{6}+m_{10}\right) y}+C_{82} e^{m_{2} y}+C_{83} e^{m_{14} y}+ \\
& \left.C_{84} e^{m_{22} y}\right) \text {. }
\end{aligned}
$$

\section{RESULTS AND DISCUSSION}

To discuss the physical significance of various parameters involved in the results, the numerical calculations have been carried out. The effects of the various parameters entering in the governing equations on the velocity, temperature and concentration are shown through graphs.

Figure 1 shows velocity profiles are depicted for various values of solutal Grashoff number Gc which is defined by the ratio of the species buoyancy force to viscous hydrodynamic force. It is noticed that when buoyancy force dominates viscous hydrodynamic force, velocity increases. Velocity profiles increases to a peak value near the plate then decreases. It can be said that velocity profiles converge pointwise. Figure 2 shows the influence of chemical reaction parameter on the velocity field. Increase in Rc results increament in velocity. Figure 3 shows velocity profiles are depicted against y for various values of Prandtl number Pr. It shows oscillatory effect. At the vicinity of the plate velocity increases with incresing values of Pr. But at certain values of y profiles intersect then leads opposite effect. In Figure 4, it is noticed that increasing values of $\mathrm{Gr}$ enhance the velocity. Figure 5 shows the oscillatory values of velocity. When $\mathrm{Q}$ increases velocity increases at first then decreases. Figure 6 shows concentration profiles are depicted for different values of Rc. It can be easily said that concentration decreases with increase in Rc. Figure 7 and Figure 8 show concentration is influenced equally by Sc and So respectively. Concentration increases with 
increasing values of these parameters near the plate. After certain value of $y$, concentration decreases when these parameters increase. Figure 9 shows temperature profiles are drawn against y for different values of Pr. It shows that values of Pr are directly proportional to temperature. In Figure 10 and Figure 11, it is noticed that temperature decreases with increasing values of $\mathrm{R}$ and $\mathrm{Q}$.

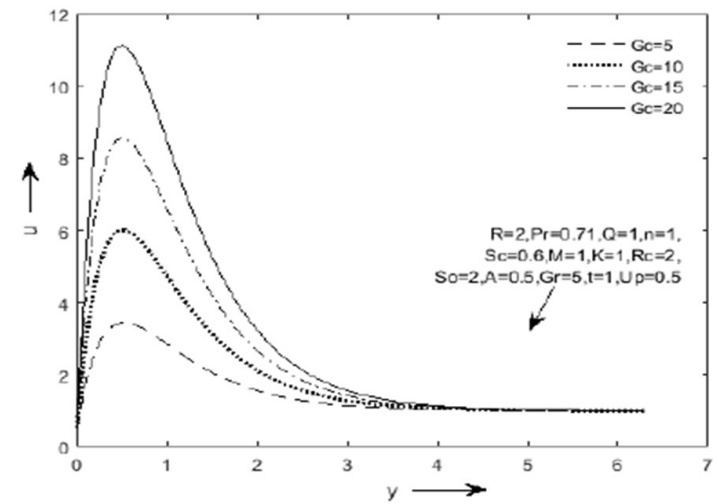

Figure 1. Velocity profiles for different values of Gc

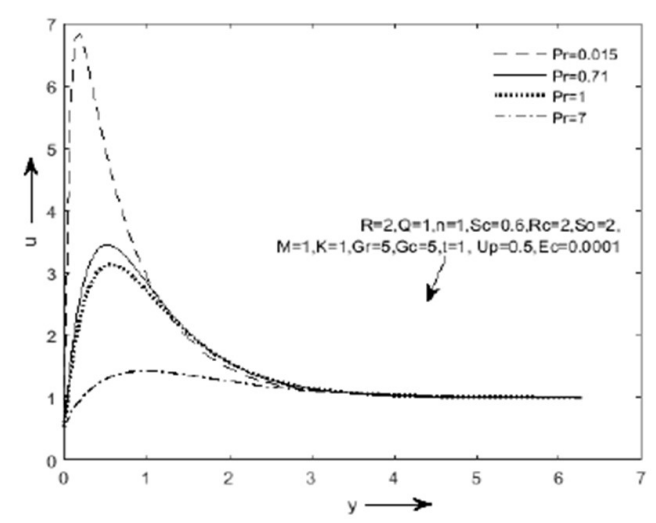

Figure 3. Velocity profiles for different values of $\mathrm{Pr}$

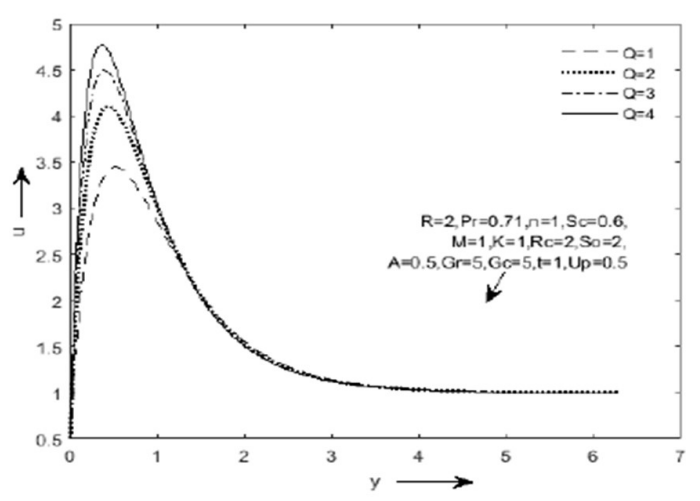

Figure 5. Velocity profiles for different values of Q

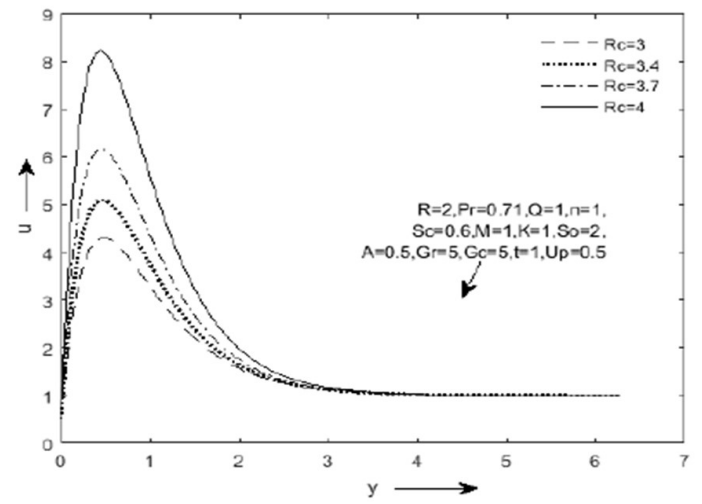

Figure 2. Velocity profiles for different values of Rc

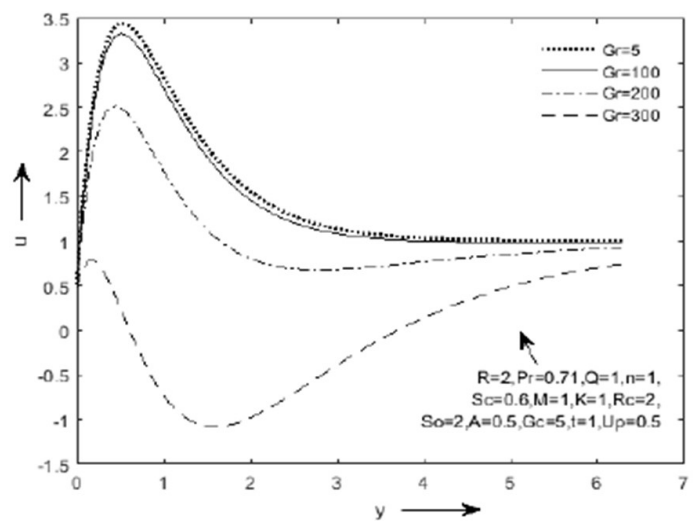

Figure 4. Velocity profiles for different values of $\mathrm{Gr}$

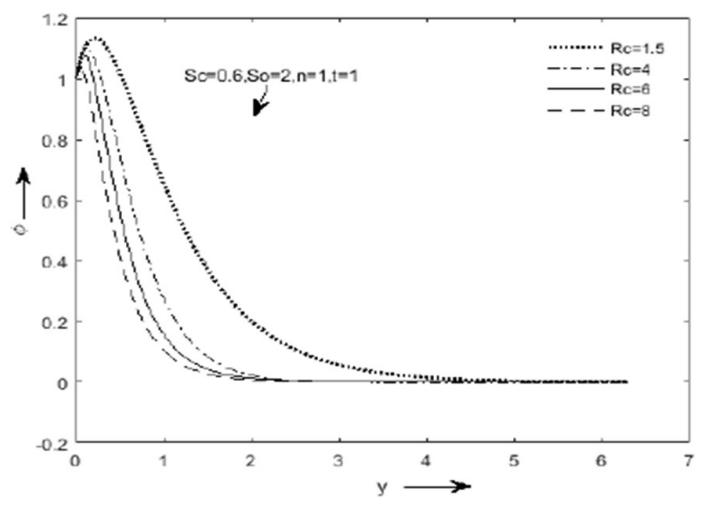

Figure 6. Concentration profiles for different values of Rc

Int. J. of Adv. in Appl. Sci. Vol. 8, No. 1, March 2019: 83 - 94 


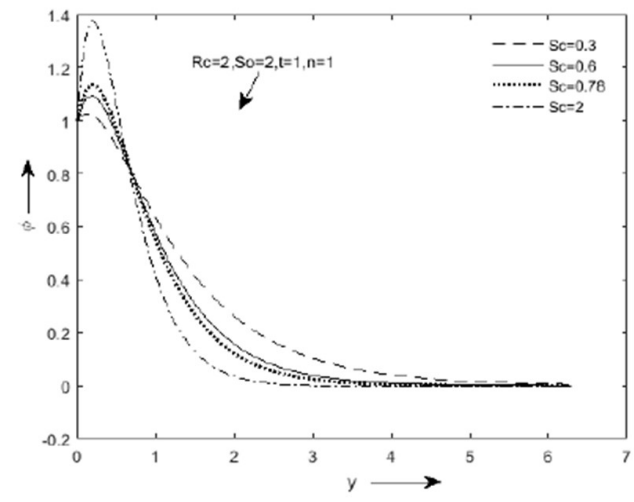

Figure 7. Concentration profiles for different values of $\mathrm{Sc}$

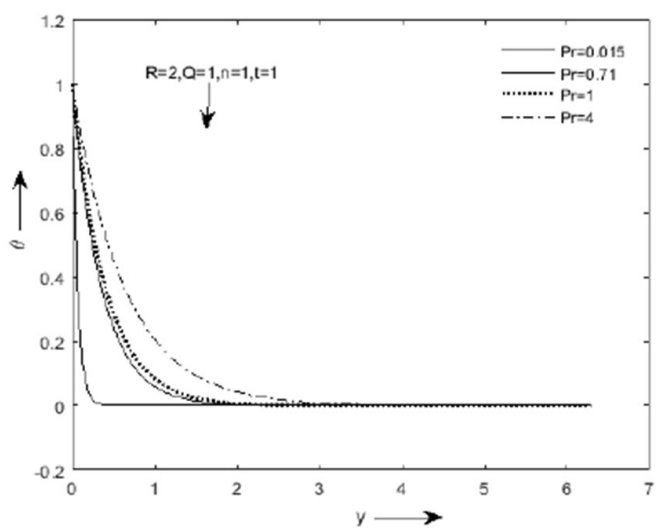

Figure 9. Temperature profiles for different valueses of $\mathrm{Pr}$

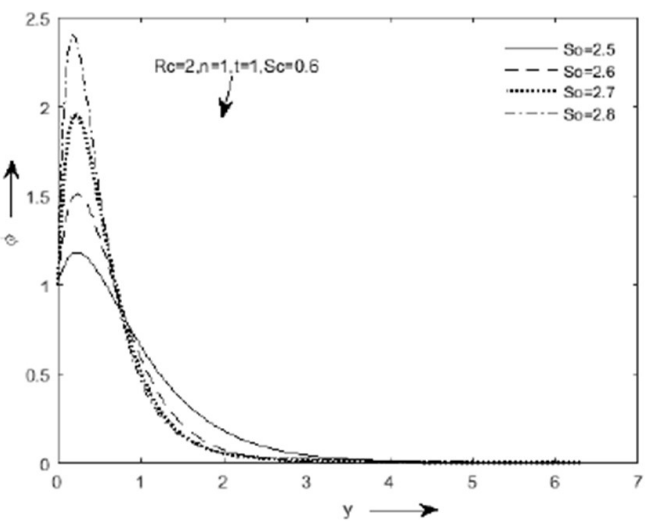

Figure 8. Concentration profiles for different values of So

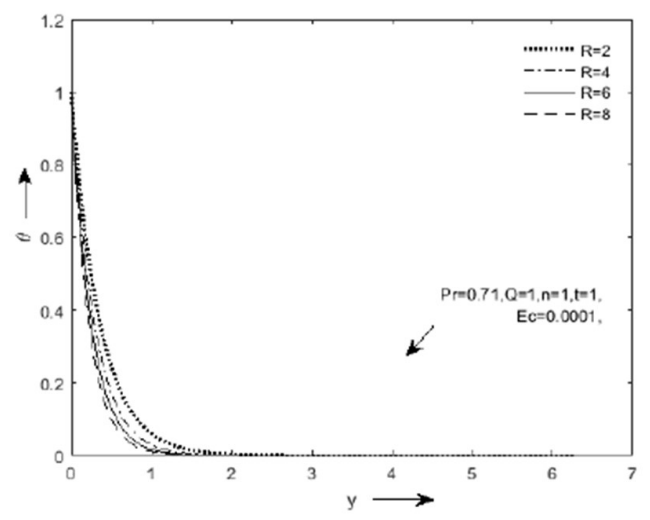

Figure 10. Temperature profiles for different valueses of $R$

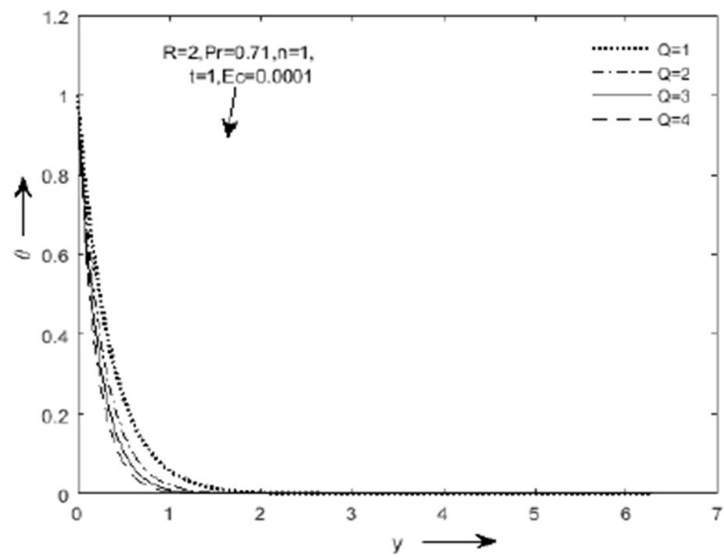

Figure 11. Temperature profiles for different valueses of Q 


\section{CONCLUSION}

Here unsteady MHD free convection flow with viscous dissipation and chemical reaction was studied. Due to addition of viscous dissipation, we find a non linearity in our energy equation. So we use Perturbation technique two times to solve the whole system. The different results are discussed through graphs. To precise the paper, the index was not included here. Finally some conclusions are drawn as follows: The parameters Gc, Gr and Rc enhance the velocity; Concentration decreases by the increament of chemical reaction parameter; The enhancing values of parameters $\mathrm{R}$ and $\mathrm{Q}$ result a decrease in temperature. But Pr shows reverse effect on temperature; In case of velocity $\operatorname{Pr}$ and $\mathrm{Q}$ show the oscillatory effect.; Similarly Sc and So shows oscillatory effect in case of concentration.

\section{REFERENCES}

[1] R. S. Sapieszko and E. Matijevi, "Preparation of well-defined colloidal particles by thermal decomposition of metal chelates. I. Iron oxides," J. Collo. Interf. Sci., vol. 74, no. 2, pp. 405-422, 1980.

[2] S. Taniguchi and A. Kikuchi, "Flow control of liquid iron by magnetic shield in a highfrequency induction furnace," Tetsu-to-Hagane, vol. 78, no. 5, pp. 753-760, 1992.

[3] C. W. Forsberg, "Hydrogen, nuclear energy and the advanced high-temperature reactor," Int. J. Hydro. Energy, vol. 28, no. 10, pp. 1073-1081, 2003.

[4] B. Yildiz and M. S. Kazimi, "Efficiency of hydrogen production systems using alternative nuclear energy technologies," Int. J. Hydro. Energy, vol. 31, no. 1, pp. 77-92, 2006.

[5] A. S. Gupta, "Steady and transient free convection of an electrically conducting fluid froma vertical plate in the presence of a magnetic field," Applied Scientific Research, vol. 9, no. 1, pp. 319-333, 1960.

[6] A. K. Singh and N. Kumar, "Free-convection flow past an exponentially accelerated vertical plate," Astrophysics and Space Science, vol. 98, no. 2, pp. 245-248, 1984.

[7] B. K. Jha, R. Prasad, and S. Rai, "Mass transfer effects on the flow past an exponentially accelerated vertical plate with constant heat flux," Astrophysics and Space Science, vol. 181, no. 1, pp. 125-134, 1991.

[8] S. F. Ahmmed, M. K. Das, and L. E. Ali, "Analytical study on unsteady MHD free convection and mass transfer flow past a vertical porous plate," American journal of applied mathematics, vol. 3, no. 2, pp. 64-74, 2015.

[9] A. J. Chamkha and A. M. Aly, "Heat and mass transfer in stagnation point flow of a polar fluid towards a stretching surface in porous media in the presence of Soret, Dufour and chemical reaction effects," Chem. Eng. Commun., vol. 198, no. 2, pp. 214-234, 2010.

[10] Aurangzaib, A. R. M. Kasim, N. F. Mohammad, and S. Shafie, "Effect of thermal stratification on MHD free convection with heat and mass transfer over an unsteady stretching surface with heat source, Hall current and chemical reaction," Int. J. Adv. Eng. Sci. Appl. Math., vol. 4, no. 3, pp. 217-225, 2012.

[11] M. Abd El-Aziz, "Effect of time-dependent chemical reaction on stagnation point flow and heat transfer over a stretching sheet in a nanofluid," Phys. Sci., vol. 89, 2014.

[12] D. Pal and G. Mandal, "Influence of thermal radiation on mixed convection heat and mass transfer stagnation-point flow in nanofluids over stretching/shrinking sheet in a porous medium with chemical reaction," Nuclear Eng. Design, vol. 273, pp. 644-652, 2014.

[13] D. Pal and H. Mondal, "MHD non-Darcian mixed convection heat and mass transfer over a non-linear stretching sheet with Soret-Dufour effects and chemical reaction," Int. Commun. Heat Mass Transf. vol. 38, pp. 463-467, 2011.

[14] Mahajan, R. L. and Gebhart, B. B., "Viscous dissipation effects in Buoyancy-Induced flows," Int. J. Heat Mass Transfer, vol. 32, no. 7, pp. 1380-1382, 1989.

[15] Israel-cookey, C., Ogulu, A., and Omubo-Pepple, V.M., "The influence of viscous dissipation and radiation on unsteady MHD free convection flow past an infinite heated vertical plate in a porous medium with time depedent suction," Int. J. Heat Mass Transfer, vol. 46, no. 13, pp. 2305-2311, 2003.

[16] J. Zueco Jordan, "Network Simulation Method Applied to Radiation and Dissikpation Effects on MHD Unsteady Free Convection over Vertical Porous Plate," Appl. Math., Modelling, vol. 31, no. 20, pp. 2019-2033, 2007.

[17] S. Suneetha, N. Bhaskar Reddy, and V. Ramachandra Prasad, "The thermal radiation effects on MHD free convection flow past an impulsively started vertical plate with variable surface temperature and concentration," Journal of Naval Architecture and Marine engineering, vol. 2, pp. 57-70, 2008.

[18] S. Suneetha, N. Bhaskar Reddy, and V. Ramachandra Prasad, "Radiation and mass transfer effects on MHD free convection flow past an impulsively started isothermal vertical plate with dissipation," Thermal Science, vol. 13, no. 2, pp. 71-181, 2009.

[19] Hitesh Kumar, "Radiative Heat Transfer with Hydro magnetic flow and viscous dissipation over a stretching surface in the presence of variable heat flux," Thermal Science, vol. 13, no. 2, pp. 163-169, 2009. 